\title{
The effects of prior inputs on auditory perceptual processing
}

\author{
CAROL BERGFELD MILLS, DAVID L. HORTON, and MICHELE L. KELLY \\ University of Maryland, College Park, Maryland 20742
}

\begin{abstract}
Two experiments are reported on subjects' ability to attend to an auditory channel (i.e., ear of input). The subjects' task was to identify the last tone (target) in a sequence of tones as "sharp" or "dull." The results indicated that when all tones of a given sequence were presented in the same ear, the target was more easily and rapidly identified than when half of the tones were randomly presented to each ear. For sequences in which half of the tones were presented to each ear, the greater the number of successive tones in the same ear as the target, the better or faster the target was identified. Differences were generally greater with a shorter intertone interval $(250 \mathrm{msec})$ than with a longer one $(600 \mathrm{msec})$. The results show that "attentional" effects can occur when prior inputs are present on a given auditory channel.
\end{abstract}

Shiffrin, Pisoni, and Castaneda-Mendez (1974) reported two identification experiments that appeared to indicate an absence of the ability to selectively attend to one of the two ears. The basic conditions involved a comparison of identification in simultaneous and sequential presentation of one of four syllables. In the sequential condition, subjects monitored first one ear and then the other in a known order for the target stimulus. The assumption here was that the subjects would always attend to one ear or the other and, if selective attention is possible, they should be able to readily identify a stimulus that was presented to the attended ear. In the simultaneous condition, subjects monitored both ears at the same time for the target stimulus. For this condition it was assumed that the subjects either would not attend to a particular ear or would have to divide attention. Thus, if selective attention is possible, performance in the simultaneous condition should be poorer than in the sequential condition. If selective attention is not possible, performance in the simultaneous and sequential conditions should be equivalent, which was the result obtained by Shiffrin et al.

Shiffrin et al. (1974) proposed that "attentional" effects arise in short-term memory. In other words, all information received at the sensory level is perceptually processed, but only that which is subsequently attended to in short-term memory is retained. According to this account, the results of experiments that appear to show selective attention (e.g., Treisman \& Riley, 1969) are attributable to high memory load demands. It is for this

The research was supported by grants from the Biomedical Sciences Support Committee, University of Maryland, to the Center for Language and Cognition. Computer services were provided by the Computer Science Center of the University of Maryland. The authors wish to acknowledge the generous assistance of Richard H. Meltzer with part of the computer programs. reason that Shiffrin et al. (1974) used a procedure that required minimal memory load.

The concern in the present research is the generality of the Shiffrin et al. (1974) findings to situations that involve more than one input to a given channel (i.e., ear). With more than one input to a given channel, attentional effects may occur even when the memory load requirements are minimal. In the present experiments, sequences of stimuli were used instead of single isolated stimuli and subjects were asked to identify only the last input of a sequence. Because only the last input had to be identified, the memory overload effects hypothesized by Shiffrin et al. should have been minimized. There were two basic conditions employed in these studies: (1) the predictable condition in which the ear of input of the last input could be anticipated by the subject, and (2) the unpredictable condition in which the ear of input of the target could not be anticipated. Unlike the Shiffrin et al. study, the stimuli used for these experiments were tones rather than synthesized speech, because it has been claimed that attentional effects should be less pronounced for tonal stimuli than for isolated syllables (Shiffrin et al., 1974).

\section{EXPERIMENT 1}

\section{Method}

The subjects' task was to identify which of two different tones was the last tone in a sequence of tones. On each trial, a sequence of tones was presented that ranged in length from 1 to 12 tones. One of two warning lights was flashed to begin each trial, indicating in which ear the sequence would begin.

Experimental design. The sequences were 1, 2, 4, 6, 8, 10, or 12 tones in length. The ear of input for one-tone sequences could always be anticipated from the warning light. The ear of arrival for two-tone sequences could never be anticipated. In this sense the one-tone sequences were considered predictable and two-tone sequences were unpredictable. For sequences longer than two tones, predictable sequences had all tones presented to the same ear and unpredictable sequences had 
half of the tones randomly presented to each ear. For these longer sequences, if the first two tones were presented to the same ear, all remaining tones were also presented to that ear (i.e., predictable). If the first two tones were presented to different ears, the sequence was unpredictable.

Materials and Apparatus. The two tones composing each sequence were a $1,000-\mathrm{Hz}$ sine wave and a $1,000-\mathrm{Hz}$ square wave, which were computer-generated. In a given sequence, the two tones were randomly generated, with the restriction (at sequence lengths of four or more tones) that each tone must occur at least one-fourth of the time.

During the experimental session, a total of 196 sequences, 98 predictable and 98 unpredictable, were presented to each subject. These sequences were generated according to a Latin square design with 14 blocks of 14 sequences. Within each block, two sequences of each length were presented, with half of the sequences being predictable and half unpredictable. Care was also taken to ensure that other sequence characteristics, such as ear of target input, were carefully balanced.

All sequences were produced such that the interval between the appropriate warning light (i.e., left or right side) and the first tone was $1 \mathrm{sec}$. The tones themselves were presented for $20 \mathrm{msec}$, with a $250-\mathrm{msec}$ intertone interval (ITI). All experimental trials took place in a background of uncorrelated white noise, which was continuously presented throughout the experiment. The tone stimuli and noise were mixed and amplified, with the tones presented at $86 \mathrm{~dB}$ SPL and the noise presented at $71 \mathrm{~dB}$ SPL through pairs of matched and calibrated Sennheiser headphones.

Procedure. The subjects were run individually in soundattenuated rooms. All stimuli were presented on-line by a PDP-12 computer. A 30 -min practice session was performed to familiarize the subjects with the tones and the task. This included training the subjects to discriminate between the two tones when presented individually and to identify the square and sine waves as "sharp" and "dull," respectively, by pressing one of two telegraph keys.

The experimental sequences were presented without prior knowledge concerning the length of any given sequence or feedback about correctness of responses. Responses were recorded by the computer. Two subjects heard the sequences in each of 14 orders of presentation.

Subjects. The subjects were 28 normal-hearing students at the University of Maryland who were enrolled in psychology classes and who participated for extra course credit. The data for seven additional subjects that clustered around the chance performance of $50 \%(45 \%-66 \%$ correct) were excluded from the analyses.

\section{Results}

The main findings from this experiment indicate that, although the difference in performance on predictable $(89.3 \%$ correct) and unpredictable ( $86.4 \%$ correct) sequences was not large, performance was superior on predictable sequences at all sequence lengths. A repeatedmeasures analysis of variance performed on number of correct responses for sequences of lengths of 1-12 tones showed this effect to be significant $[\mathrm{F}(1,27)=7.13$, $\mathrm{p}<.025, \mathrm{MSe}=1.85]$. Neither sequence length nor the interaction between sequence length and type of sequence approached significance.

A separate analysis was performed on the number of correct responses for sequences of one and two tones. It will be recalled that one-tone sequences were always predictable from the warning light and two-tone sequences were always unpredictable. Although performance on
Table 1

Percent Correct Responses (PC) for Different Run Lengths in Unpredictable Sequences of 6, 8, 10, and 12 Tones Combined in Experiment 1

\begin{tabular}{|c|c|c|c|c|c|c|c|c|c|}
\hline \multicolumn{10}{|c|}{ Run Length } \\
\hline \multicolumn{2}{|c|}{1} & \multicolumn{2}{|c|}{2} & \multicolumn{2}{|c|}{3} & \multicolumn{2}{|c|}{4} & \multicolumn{2}{|l|}{5} \\
\hline PC & $\mathrm{N}$ & PC & $\mathrm{N}$ & PC & $\mathrm{N}$ & PC & $\mathrm{N}$ & PC & $\mathrm{N}$ \\
\hline 85.6 & 16 & 86.4 & 20 & 86.6 & 10 & 90.3 & 8 & 94.6 & 2 \\
\hline
\end{tabular}

Note $-N=$ number of sequences of a given run length each subject heard.

one-tone sequences ( $88.0 \%$ correct) was better than that on two-tone sequences ( $84.6 \%$ correct), the difference was not significant, which is consistent with the Shiffrin et al. (1974) findings. However, a further analysis compared performance on two-tone sequences with both tones in the same ear $(86.8 \%$ correct) with those sequences containing tones alternating between the ears $(82.5 \%$ correct). A one-tailed $t$ test indicated that performance was significantly better when both tones were presented to the same ear $[\mathrm{t}(27)=1.70, \mathrm{p}<.05]$. This finding suggests that identification performance is better when there are prior inputs on a given auditory channel, because for two-tone sequences no prior knowledge was available to predict ear of input.

If performance is better when there is a prior input in a given ear, then it might be expected that the more tones presented in succession to the target ear, the better performance should be. Thus, the data of the longer unpredictable sequences were examined in terms of the number of consecutive tones on the same ear as the target tone (i.e., run length). For example, if the last three tones of a sequence were presented to the right ear and the preceding tone to the left ear, the run length would be three. The data for sequence lengths of 6-12 tones were combined in this analysis.

The effect of run length is shown in Table 1. There was a steady increase in identification performance as the number of consecutive prior tones presented to the same ear as the target tone increased. An analysis of variance indicated that this effect was significant $\left[\mathrm{F}(4,108)=2.81, \mathrm{p}<.05, \mathrm{MSe}=142.2 ; \mathrm{F}_{\max }(5,27)=\right.$ 1.72]. A further analysis indicated that the linear trend was also significant.

\section{EXPERIMENT 2}

The purpose of this experiment was both to replicate and to extend the findings of Experiment 1 . Because the results of that experiment were of relatively small magnitude, a more sensitive response measure, reaction time (RT), was used in addition to the number of correct responses.

A between-subjects factor, ITI, was also introduced in this experiment. The ITIs used were $250 \mathrm{msec}$ and $600 \mathrm{msec}$. ITI was used as a factor because introspective 
observations with our tone stimuli, as well as previous research (cf. Fraisse, 1963; Garner \& Gottwald, 1968) suggested that as the interval between successive stimuli increases, the sequence of stimuli gives rise to perceptions that are qualitatively different. For example, Garner and Gottwald (1968) stated that with rates of presentation faster than two elements/sec, the phenomenal experience is that of an integrated sequence, whereas at slower rates, the experience is that of an "unintegrated series of single elements." Thus, an ITI of $250 \mathrm{msec}$ should result in more integration of the sequence (i.e., greater perceived temporal coherence) than that of $600 \mathrm{msec}$. As a result, prior inputs may have more effect on performance at a $250-\mathrm{msec}$ ITI than at a $600-\mathrm{msec}$ ITI and the difference in performance between predictable and unpredictable sequences should be greater with an ITI of $250 \mathrm{msec}$.

\section{Method}

Experimental design. The design of this experiment was the same as that of Experiment 1, except that RTs were collected along with identification responses. Also, two groups of subjects were employed, one given sequences having $250-\mathrm{msec}$ ITI and the other given sequences having a $600-\mathrm{msec}$ ITI.

Materials and Apparatus. Only a few changes in the previously described stimuli were made for this experiment. The white-noise level was lowered to $65 \mathrm{~dB}$ SPL so that identification performance would be higher than in Experiment 1 in order to minimize speed-accuracy tradeoff. In addition, some run lengths were modified in unpredictable sequences so that more sequences with a run length of five could be presented. To allow direct comparisons to be made between RTs in the $600-$ and $250-\mathrm{msec}$ ITI conditions, RT was calculated from the end of the appropriate ITI following the last tone of a given sequence.

Procedure. The proceudre was the same as that for Experiment 1 , with the addition of RT instructions that emphasized both speed and accuracy.

Subjects. The subjects were 56 normalhearing students at the University of Maryland who were enrolled in psychology classes and who participated for course credit. The data from 20 additional subjects were omitted from the analyses because of relatively poor performance (either a relatively high error rate, $40 \%-70 \%$ correct, or extremely slow average RTs, greater than $1,000 \mathrm{msec})^{1}$

\section{Results}

The data for percent correct identifications are summarized in Table 2. As can be seen, performance on predictable sequences was better than that on unpredictable sequences. An analyses of variance indicated that for all sequence lengths combined, this difference was significant $[F(1,54)=12.64, p<.001, \mathrm{MSe}=.61]$. As in Experiment 1, the advantage for predictable sequences held at all sequence lengths. This analysis also showed a significant advantage for sequences presented at an ITI of $600 \mathrm{msec}$ compared to $250 \mathrm{msec}[\mathrm{F}(1,54)=6.13$, $\mathrm{p}<.05, \mathrm{MSe}=11.65]$. Type of sequence and ITI also showed a significant interaction $[\mathrm{F}(1,54)=4.51, \mathrm{p}<.05$, $\mathrm{MSe}=.61]$. Newman-Keuls tests were performed to compare the means for the four conditions. These comparisons were all significant $(p<.001)$, except for the comparison between predictable and unpredictable sequences at an ITI of 600 msec.
Table 2

Mean Percent Correct and Mean Reaction Time (in Milliseconds) for Correct Responses for All Length Sequences Combined in Experiment 2

\begin{tabular}{cccccccc}
\hline & \multicolumn{6}{c}{ Type of Sequence } \\
\cline { 2 - 4 } \cline { 6 - 8 } Intertone & \multicolumn{3}{c}{ Percent Correct } & & \multicolumn{3}{c}{ Reaction Time } \\
\cline { 2 - 4 } \cline { 6 - 8 } Interval* & $\mathrm{P}$ & $\mathrm{U}$ & Total & & $\mathrm{P}$ & $\mathrm{U}$ & Total \\
\hline 250 & 91.5 & 89.0 & 90.2 & & 700 & 743 & 721 \\
600 & 95.2 & 94.6 & 94.9 & & 684 & 698 & 691 \\
Total & 93.4 & 91.8 & & & 692 & 721 & \\
\hline
\end{tabular}

Note-P = predictable; $U=$ unpredictable.

*In milliseconds.

The RT results, which are also shown in Table 2, showed faster RTs for predictable than for unpredictable sequences. An analysis of variance indicated that this difference was significant $[F(1,54)=13.74, p<.001$, $\mathrm{MSe}=1,029.74]$. Again, performance was superior for predictable sequences at all sequence lengths. In this analysis, the only other significant effect was that of sequence length $[F(5,270)=46.38, p<.001, \mathrm{MSe}=$ 780.71]. The mean RT for sequence lengths of 1 or 2 , $4,6,8,10$, and 12 were $783,736,718,660,657$, $656 \mathrm{msec}$, respectively.

As in Experiment 1, separate analyses were performed on sequences of one and two tones. Analyses were performed for both correct identifications and for RT. Neither analysis indicated any significant main effects or interactions. An additional test was performed on correct identifications with two-tone sequences. For an ITI of $250 \mathrm{msec}$, performance was superior when both tones were presented to the same ear (90.5\% correct) than when they were presented to different ears $(85.2 \%$ correct) $[\mathrm{t}(27)=1.93, \mathrm{p}<.05$, one-tailed] . As expected, the difference between the comparable means for an ITI of $600 \mathrm{msec}$ (91.8\% for different ears and $92.6 \%$ for same ear) was not significant. RT data showed the same pattern of results as the correct identification results and will be presented later.

An analysis of identification performance in Experiment 2 did not reveal any significant effect of run length. It is possible that the absence of a run-length effect in this case was due to a ceiling effect, since in this experiment the identification task was intended to be easier and was easier. This interpretation is supported by the results of the RT analysis for unpredictable sequences.

The RT data for correct responses only are presented for the two ITIs combined in Table 3. Run length was analyzed separately for each sequence length, because length of sequence was shown to produce a significant effect in the overall RT analyses. For each of the five sequence lengths, an analysis of variance was performed with ITI and run length as factors. The $F$ values for run length are given in Table 3 , as well. As seen there, run length was significant for sequences 2,10 , and 12 tones in length. ITI was not a significant factor in these analyses, nor did ITI interact with run length except for two-tone sequences. For the twotone sequences, a Newman-Keuls test revealed that for 
Table 3

Mean Reaction Time (in Milliseconds) for Correct Responses in Unpredictable Sequences as a Function of Length of Sequence and Run Length in Experiment 2

\begin{tabular}{|c|c|c|c|c|c|c|c|c|c|c|c|c|}
\hline \multirow{3}{*}{$\begin{array}{l}\text { Sequence } \\
\text { Length }\end{array}$} & \multicolumn{10}{|c|}{ Run Length } & \multirow{2}{*}{\multicolumn{2}{|c|}{$\begin{array}{l}\text { Run Length Factor in } \\
\text { Analyses of Variance }\end{array}$}} \\
\hline & \multicolumn{2}{|c|}{1} & \multicolumn{2}{|c|}{2} & \multicolumn{2}{|c|}{3} & \multicolumn{2}{|c|}{4} & \multicolumn{2}{|c|}{5} & & \\
\hline & RT & $\mathrm{N}$ & RT & $\mathrm{N}$ & RT & $\mathrm{N}$ & $\mathrm{RT}$ & $\mathrm{N}$ & RT & $\mathrm{N}$ & $\mathbf{F}$ & df \\
\hline 2 & 830 & 14 & 751 & 14 & & & & & & & $13.94 \dagger$ & 1,54 \\
\hline 6 & 749 & 7 & 723 & 7 & & & & & & & 1.52 & 1,54 \\
\hline 8 & 697 & 4 & 674 & 5 & 638 & 5 & & & & & 2.97 & 2,108 \\
\hline 10 & 757 & 3 & 673 & 4 & 658 & 3 & 616 & 4 & & & $6.52 \dagger$ & 3,162 \\
\hline 12 & 706 & 2 & 709 & 3 & 622 & 2 & 599 & 3 & 653 & 4 & $4.70^{*}$ & 4,216 \\
\hline
\end{tabular}

Note $-N=$ number of sequences of a given type each subject heard. $\quad{ }^{*} p<.01 . \quad \dagger p<.001$.

the 250 -msec ITI, a run length of one $(887 \mathrm{msec})$ was slower than for a run length of two $(755 \mathrm{msec})(\mathrm{p}<.01)$. For the 600-msec ITI, there was no significant difference between run lengths of one and two $(773$ vs. $747 \mathrm{msec}$, respectively). Linear trends were also found to be significant for sequence lengths of 8,10 , and 12 . Thus, the general trend was for faster RTs to occur as run length increased.

\section{DISCUSSION}

The present experiments showed that for the correct identification data, predictable sequences (all same ear) lead to better performance than do unpredictable sequences (random to different ears) for all sequence lengths. In addition, the RT analysis from Experiment 2, also showed faster RT for predictable sequences than for unpredictable sequences for all sequence lengths. A comparison of one- and two-tone sequences (the conditions most comparable to the conditions in Shiffrin et al., 1974) showed no differences. However, for the two-tone sequences, performance was better when both tones were presented to the same ear than when they were presented first to one ear and then to the other ear. Since for two-tone sequences no prior knowledge was available, the results clearly demonstrate that prior inputs alone can influence performance. In addition, in the first experiment, identification performance increased as a function of run length (number of consecutive tones on same ear as the target tone) in a linear manner. In the second experiment, run length generally had a significant linear effect on RT, with RT becoming faster as run length increased. These results show that attentional effects occur when prior inputs are in the same channel as the target.

Support for the notion of perceived temporal coherence was found in the second experiment, in which predictable sequences resulted in better identification performance than did unpredictable sequences for the 250-msec ITI (higher temporal coherence), but not for the $600-\mathrm{msec}$ ITI. A similar pattern of results appeared for the analysis of two-tone sequences for RT. The results showing overall poorer performance with the 250 -msec ITI than with the 600 -msec ITI may suggest that with greater perceived temporal coherence, the target tone is more difficult to separate from the remainder of the sequence.

The findings reported here are consistent with those of other studies in which sequences of tones or clicks were presented in either a monaural or a systematically alternating, interaural manner (e.g., Massaro, 1976). These studies have found, for example, less performance accuracy for interaural sequences than for monaural ones.

Shiffrin et al. (1974), as well as Shiffrin and Schneider (1977), attributed the results of most studies showing attentional effects to a memory load factor. The results of the present studies, however, are not subject to that explanation, since our subjects only had to identify one tone per sequence (the last tone). Our data suggest that there were no memory load effects; identification performance was at least as good for any given sequence length as it was for the isolated single tone.

In summary, the results of the present studies indicate that the perceiver can perform better when there are immediate prior inputs to the same ear as the target. They suggest that experiments using isolated stimuli may not reveal what the perceiver is capable of doing when prior inputs are present on a given auditory channel.

\section{REFERENCES}

Fraisse, P. The psychology of time. New York: Harper \& Row, 1963.

Garner, W. R., \& GotTwald, R. L. The perception and learning of temporal patterns. Quarterly Journal of Experimental Psychology, 1968, 20, 97-109.

Massaro, D. W. Perceiving and counting sounds. Journal of Experimental Psychology: Human Perception and Performance, 1976, 2, 337-346.

Shiffrin, R. M., Pisoni, D. B., \& Castaneda-Mendez, K. Is attention shared between the ears? Cognitive Psychology, 1974, 6, 190-215.

Shiffrin, R. M., \& Schneider, W. Controlled and automatic human information processing: II. Perceptual learning, automatic attending, and a general theory. Psychological Review, 1977, 84, 127-190.

Treisman, A. M., \& Riley, J. G. Is selective attention selective perception or selective response? A further test. Journal of Experimental Psychology, 1969, 79, 27-34.

\section{NOTE}

1. More subjects were omitted from the 250 -msec ITI condition (the more difficult ITI condition) than from the $600-\mathrm{msec}$ ITI condition. As a result, the differences between the $250-\mathrm{msec}$ and $600-\mathrm{msec}$ ITIs were reduced.

(Received for publication July 19, 1982.) 